$17^{\text {th }}$ International Congress of Metrology, 13012 (2015)

DOI: $10.1051 /$ metrology / 201513012

(C) Owned by the authors, published by EDP Sciences, 2015

\title{
Fast checking of CMM geometry with a patented tool
}

\author{
Jean-François Manlay ${ }^{1}$ \\ ${ }^{1}$ CEA/DAM/Gramat, F-46 500 Gramat, France
}

\begin{abstract}
Résumé. L'utilisation d'une Machine à Mesurer Tridimensionnelle (MMT) requiert une bonne connaissance des défauts géométriques de la machine, afin d'obtenir des résultats fiables. Les essais de réception ou l'autocontrôle à partir de la série de normes ISO 10360 sont suffisants dans certains cas, mais ne permettent pas, en cas d'erreurs de mesure, de remonter au paramètre géométrique défaillant.

Un nouvel outil, construit à partir d'éléments étalonnés, permet d'obtenir une information globale sur la géométrie de la MMT, et ne nécessite que peu de temps de mesure pour réaliser une image des défauts géométriques de la MMT. Nous présentons ce nouvel outil, breveté, ainsi qu'un exemple d'utilisation.
\end{abstract}

\section{Introduction}

Acceptance tests or recertification tests based on ISO 10360 standards [1] give results which must be within a tolerance to pass the test. If some results are out of tolerance, the CMM has to be calibrated. This operation can take a long time, because the cause of the defect is generally unknown.

A ball bar test [2] [3] can give some information about rotations of the CMM, but it is time consuming, and the result isn't linked to the meter definition.

In fact, the new tool presented here resolves those little defects, and facilitates the wrong parameter identification, so that the calibration required is less demanding than a total calibration.

Starting from geometrical defects of a CMM, we present this new tool and the results of a demonstration.

\section{Retrieval about CMM geometry}

Geometrical defects generated by the CMM manufacturing are compensated with a matrix, which is usually built with an interferometer and its reflectors. The real displacements of the CMM are measured with the interferometer, and the difference between the CMM readings and the interferometer ones are included in the compensation matrix, so that the measurements can be accurate. Rotations during displacements can be measured with an electronic level.

Along each axis, the matrix compensates the trueness of the scale, the straightness of the displacement perpendicularly of it (which are linear parameters) and the roll, the pitch and the yaw (which are angular parameters of rotation) (fig. 1).

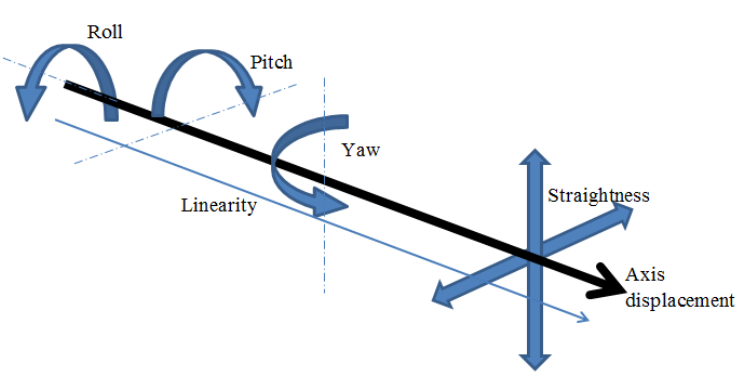

Figure 1: defects along an axis

The matrix contains the compensation values measured along each axis with a step (fig.2). The CMM interpolates its position from a math function between steps, so that each measured point can be compensated.

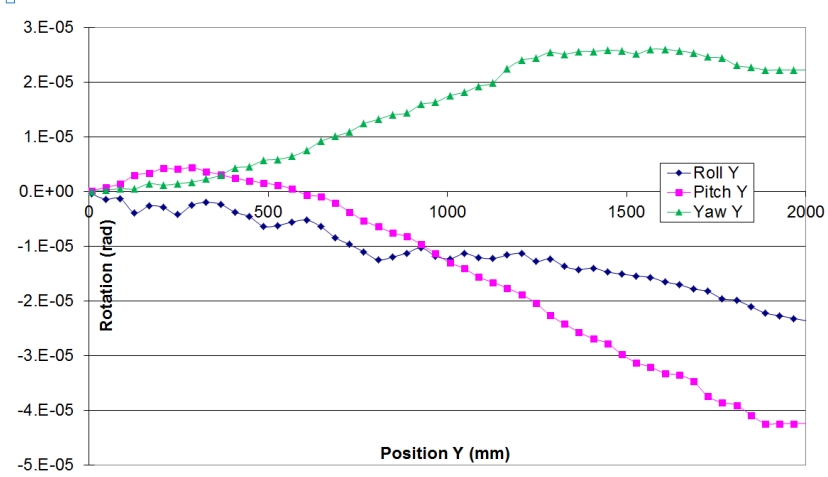

Figure 2 : graphical representation of the $\mathrm{Y}$ axis compensation values (rotations only)

In addition to those, the three values of perpendicularity between axes, two by two, are included in the matrix (fig.3). 


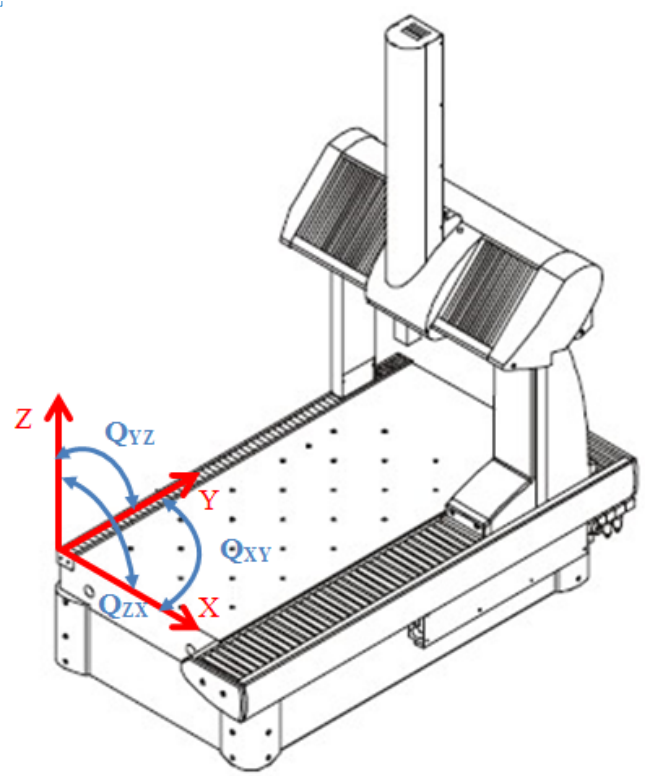

Figure 3: axes and perpendicularity

Those values are considered as constant values, they can be integrated in rotation values or calculated as straightness defect, and add to a linear compensation, it depends on the CMMs manufacturer. All those compensated values are often called " 21 parameters matrix". Each term of the matrix is really accurate at the moment of matrix creation. All temperature variations, vibrations or probe collisions (...) can alter CMM geometry, without any change on the matrix. For this reason, it's essential to check regularly the compensation validity.

The compensation of geometrical defects during the measurement is calculated from the method of small displacements torsor [4]. During the movement of an axis, the two others axes are considered as fixed. This allows writing the geometrical defect equation as the sum of defects from each axis.

In our case (fig. 4), the calculation begins by the displacement of the $\mathrm{Y}$ axis, then the $\mathrm{X}$ axis and then the $\mathrm{Z}$ axis:

$$
\begin{aligned}
& \overrightarrow{E_{Y}(P)}=\overrightarrow{E_{Y}\left(O_{1}\right)}+\overrightarrow{W_{Y}} \wedge \overrightarrow{O_{1} P} \\
& \overrightarrow{E_{X}(P)}=\overrightarrow{E_{X}\left(O_{2}\right)}+\overrightarrow{W_{X}} \wedge \overrightarrow{O_{2} P} \\
& \overrightarrow{E_{Z}(P)}=\overrightarrow{E_{Z}\left(O_{3}\right)}+\overrightarrow{W_{Z}} \wedge \overrightarrow{O_{3} P}
\end{aligned}
$$

$\overrightarrow{E_{l}\left(O_{J}\right)}$ represents linear defects of the $i$ axis, noted $L_{i x}$, $L_{i y}, L_{i z}$ afterward, and $\vec{W}_{l}$ represents rotation defects $R_{i x}$, $R_{i y}, R_{i z}$ (fig. 5).

$\overrightarrow{O_{J} P}$ is the vector between a particular point of the $j$ axis and the probe center ball, so it's the sum of scale readings $\left(P_{X}, P_{Y}, P_{Z}\right)$ and probe offsets $\left(T_{X}, T_{Y}, T_{Z}\right)$.

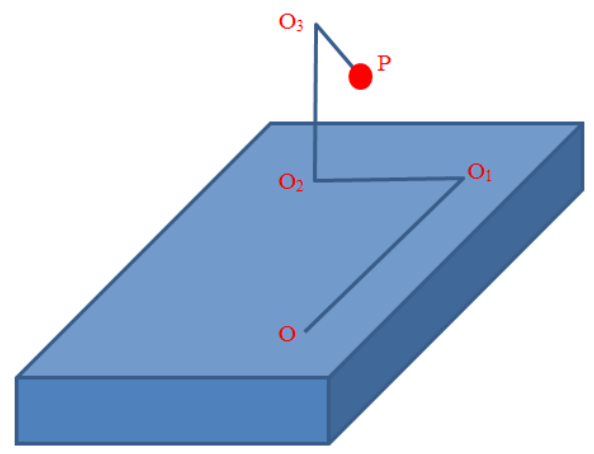

Figure 4: moves representation

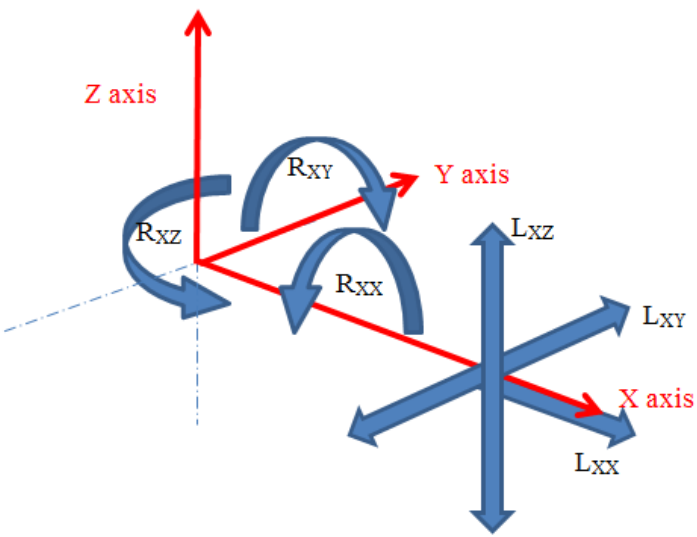

Figure 5: defect explanation along the $X$ axis

Those cross products give the following contribution of each axis displacement.

- Contribution of the Y axis displacement

$$
\begin{aligned}
\overrightarrow{E_{Y}(P)} & =\left[L_{Y X}+R_{Y Y} \cdot\left(P_{Z}+T_{Z}\right)-R_{Y Z} T_{Y}\right] \vec{X}+\left[L_{Y Y}\right. \\
& \left.+R_{Y Z} \cdot\left(P_{X}+T_{X}\right)-R_{Y X}\left(P_{Z}+T_{Z}\right)\right] \vec{Y} \\
& +\left[L_{Y Z}+R_{Y X} \cdot T_{Y}-R_{Y Y}\left(P_{X}+T_{X}\right)\right] \vec{Z}
\end{aligned}
$$

- Contribution of the $\mathrm{X}$ axis displacement

$$
\begin{gathered}
\overrightarrow{E_{X}(P)}=\left[L_{X X}+R_{X Y} \cdot\left(P_{Z}+T_{Z}\right)-R_{X Z} T_{Y}\right] \vec{X} \\
+\left[L_{X Y}+R_{X Z} T_{X} \cdot-R_{X X}\left(P_{Z}+T_{Z}\right)\right] \vec{Y} \\
+\left[L_{X Z}+R_{X X} \cdot T_{Y}-R_{X Y} T_{X}\right] \vec{Z}
\end{gathered}
$$

- Contribution of the $\mathrm{Z}$ axis displacement

$$
\begin{aligned}
& \overrightarrow{E_{Z}(P)}=\left[L_{Z X}+R_{Z Y} \cdot T_{Z}-R_{Z Z} T_{Y}\right] \vec{X}+\left[L_{Z Y}+R_{Z Z} \cdot T_{X}\right. \\
& \left.\quad-R_{Z X} T_{Z}\right] \vec{Y}+\left[L_{Z Z}+R_{Z X} \cdot T_{Y}-R_{Z Y} T_{X}\right] \vec{Z}
\end{aligned}
$$

From those three equations, the probe position is compensated along each axis using those parameters:

$$
\begin{gathered}
C_{X}=L_{X X}+L_{Y X}+L_{Z X}+R_{Y Y} \cdot\left(P_{Z}+T_{Z}\right) \\
-R_{Y Z} T_{Y}+R_{X Y} \cdot\left(P_{Z}+T_{Z}\right)-R_{X Z} T_{Y}+R_{Z Y} \cdot T_{Z}-R_{Z Z} T_{Y} \\
C_{Y}=L_{X Y}+L_{Y Y}+L_{Z Y}+R_{Y Z} \cdot\left(P_{X}+T_{X}\right)-R_{Y X}\left(P_{Z}+T_{Z}\right) \\
+R_{X Z} T_{X} \cdot-R_{X X}\left(P_{Z}+T_{Z}\right)+R_{Z Z} \cdot T_{X}-R_{Z X} T_{Z}
\end{gathered}
$$




$$
\begin{aligned}
C_{Z}= & L_{X Z}+L_{Y Z}+L_{Z Z}+R_{Y X} \cdot T_{Y}-R_{Y Y}\left(P_{X}+T_{X}\right) \\
& +R_{X X} \cdot T_{Y}-R_{X Y} T_{X}+R_{Z X} \cdot T_{Y}-R_{Z Y} T_{X}
\end{aligned}
$$

The perpendicularity between:

- $\quad \mathrm{X}$ and $\mathrm{Z}$ is included in the straightness $L_{Z X}$,

- $\quad \mathrm{X}$ and $\mathrm{Y}$ is included in the straightness $L_{X Y}$,

- $\quad \mathrm{Y}$ and $\mathrm{Z}$ is included in the straightness $L_{Z Y}$.

It can be noted that:

- $\quad L_{X X}, L_{X Y}, L_{X Z}, R_{X X}, R_{X Y}, R_{X Z}$ depend only on the X axis displacement,

- $\quad L_{Y X}, L_{Y Y}, L_{Y Z}, R_{Y X}, R_{Y Y}, R_{Y Z}$ depend only on the Y axis displacement,

- $\quad L_{Z X}, L_{Z Y}, L_{Z Z}, R_{Z X}, R_{Z Y}, R_{Z Z}$ depend only on the $\mathrm{Z}$ axis displacement.

\section{Description of the patented new tool}

Checking the CMM geometry needs to measure some known objects, linked to the meter definition, so that each parameter can be verified. It can be done with an interferometer, which is a long and expensive method, or with a step gauge, which is also long and expensive [5].

In order to check the CMM in a short time, we propose a gauge assembly, constructed with block gauges and cylinder gauges, in the shape of a triangle [6] (fig.6). Each block gauge has contact with a cylinder at each end, so the length of each side of the triangle is known, with a calculated uncertainty. Angles can be calculated by trigonometry, the uncertainty on angles is calculated by the Monte-Carlo method. This tool allows only a global check of linear and rotation defects, no local ones. Its assembly is totally described in the patent [6].

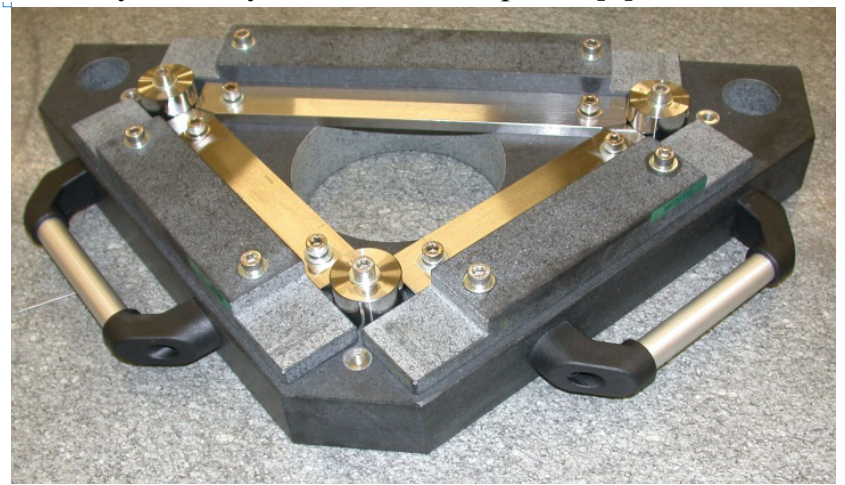

Figure 6: two-dimensional standard

\section{Calculation of residual defects using the standard}

The assembly can be measured horizontally or vertically, the hypotenuse parallel to a CMM axis. The first step is a plane measurement, to define the level for circles measurements. Then, circles can be measured, and distance between them calculated, and compared to the nominal length.

In order to demonstrate the check, we propose some hypotheses:

- If the measurement is done horizontally, the probe axis is vertical. In this case, we consider that $\mathrm{T}_{\mathrm{X}}$ and $\mathrm{T}_{\mathrm{Y}}$ are null.
- If the measurement is done vertically, the probe axis is horizontal. In this case, we consider that $T_{Z}$ is null, and one of $T_{X}$ and $T_{Y}$ can be null.

\subsection{Linear measurements in the $X Y$ plane}

This application is done with the hypotenuse parallel to the $\mathrm{X}$ axis (fig. 7). In this case, the height of the triangle (normal to the hypotenuse, by definition) is parallel to the $\mathrm{Y}$ axis, and both others sides are along diagonals.

Equation $C_{X}$ becomes:

$$
C_{X}=L_{X X}+L_{Y X}+L_{Z X}+R_{Y Y} \cdot\left(P_{Z}+T_{Z}\right)+R_{X Y} \cdot\left(P_{Z}+T_{Z}\right)
$$$$
+R_{Z Y} \cdot T_{Z}
$$

The distance between two points $\mathrm{P} 1$ and $\mathrm{P} 2$ can be calculated from:

$$
\overrightarrow{P_{1} P_{2}}=\overrightarrow{P_{1} O}+\overrightarrow{O P_{2}}=\overrightarrow{O P_{2}}-\overrightarrow{O P_{1}}
$$

The compensation between both points is the difference of compensations at each point. The difference between measured values and standard values can be assimilated as a compensation defect, which can be calculated.

The compensation of a distance between two circle centers aligned along the $\mathrm{X}$ axis, $\left(\mathrm{C}_{\mathrm{X} 2}-\mathrm{C}_{\mathrm{X} 1}\right)$, allows to eliminate values which don't depend on $\mathrm{X}$ displacement, so :

$$
\begin{aligned}
& C_{X 2}-C_{X 1}=L_{X X}\left\{X_{2}\right\}-L_{X X}\left\{X_{1}\right\} \\
& +\left(R_{X Y}\left\{X_{2}\right\}-R_{X Y}\left\{X_{1}\right\}\right)\left(P_{Z}+T_{Z}\right)
\end{aligned}
$$

It represents the trueness defect on the distance between $X_{1}$ and $X_{2}$, in addition to the $\mathrm{X}$ pitch effect.

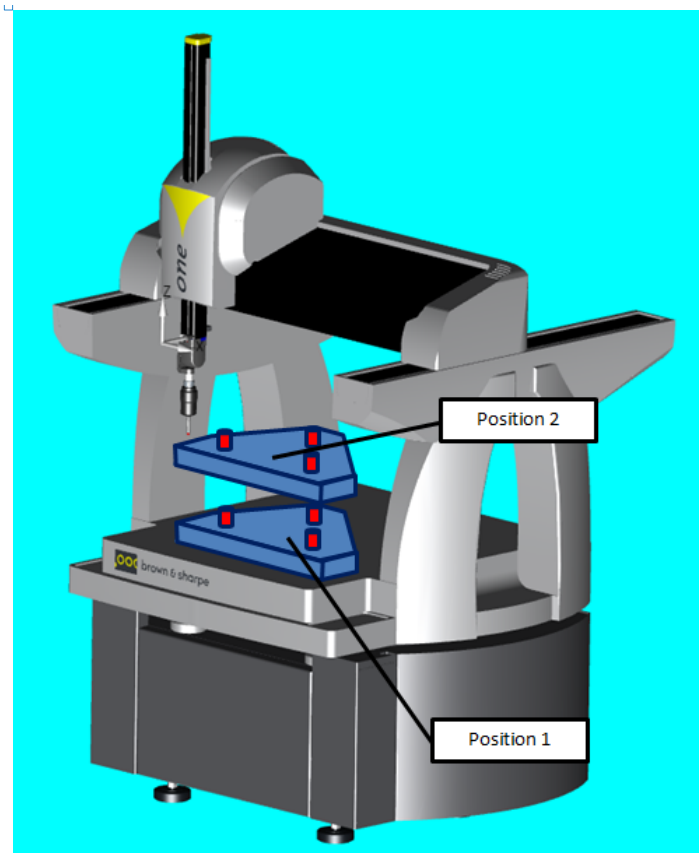

Figure 7: Positioning on the XY plane

If a second position is measured, higher than the first one, but aligned on it, the trueness defect is eliminated, and the pitch difference has the same value in both positions, then the calculation becomes:

$$
\begin{gathered}
\left(C_{X 2}-C_{X 1}\right)-\left(C_{X 4}-C_{X 3}\right) \\
=\left(R_{X Y}\left\{X_{2}\right\}-R_{X Y}\left\{X_{1}\right\}\right)\left(P_{Z 2}-P_{Z 1}\right)
\end{gathered}
$$


It represents the $\mathrm{X}$ pitch defect on a height of $\left(Z_{2}-Z_{1}\right)$. It allows also calculating the trueness of the $\mathrm{X}$ axis.

Along the $\mathrm{Y}$ axis, the method is the same. The greatest difference is the calculation along the height of the triangle, which is a calculated value, instead of a measured one.

Equation $C_{Y}$ becomes:

$C_{Y}=L_{X Y}+L_{Y Y}+L_{Z Y}+R_{Y Z} \cdot P_{X}-R_{Y X}\left(P_{Z}\right.$
$\left.+T_{Z}\right)-R_{X X}\left(P_{Z}+T_{Z}\right)-R_{Z X} T_{Z}$

Then the compensation on the height is:

$\left(C_{Y 2}-C_{Y 1}\right)=L_{Y Y}\left\{Y_{2}\right\}-L_{Y Y}\left\{Y_{1}\right\}$
$+\left(R_{Y Z}\left\{Y_{2}\right\}-R_{Y Z}\left\{Y_{1}\right\}\right) P_{X}-\left(R_{Y X}\left\{Y_{2}\right\}-R_{Y X}\left\{Y_{1}\right\}\right)\left(P_{Z}+T_{Z}\right)$

This is the sum of trueness defect along the $\mathrm{Y}$ axis on the height, with the effects of the Y yaw and the Y pitch.

With the second position, the calculation is:

$\left(C_{Y 2}-C_{Y 1}\right)-\left(C_{Y 4}-C_{Y 3}\right)$
$=-\left(R_{Y X}\left\{Y_{2}\right\}-R_{Y X}\left\{Y_{1}\right\}\right)\left(P_{Z 2}-P_{Z 1}\right)$

It allows calculating the $\mathrm{Y}$ pitch.

\subsection{Linear measurements in the $Y Z$ plane}

In this case, the standard is positioned vertically, the hypotenuse parallel to the $\mathrm{Y}$ axis. The height of the triangle is parallel to the $\mathrm{Z}$ axis. Measurements must be done on the left side and on the right side of the CMM, without any rotation of the head between both sides. The probing system is parallel to the $\mathrm{X}$ axis (fig. 8).

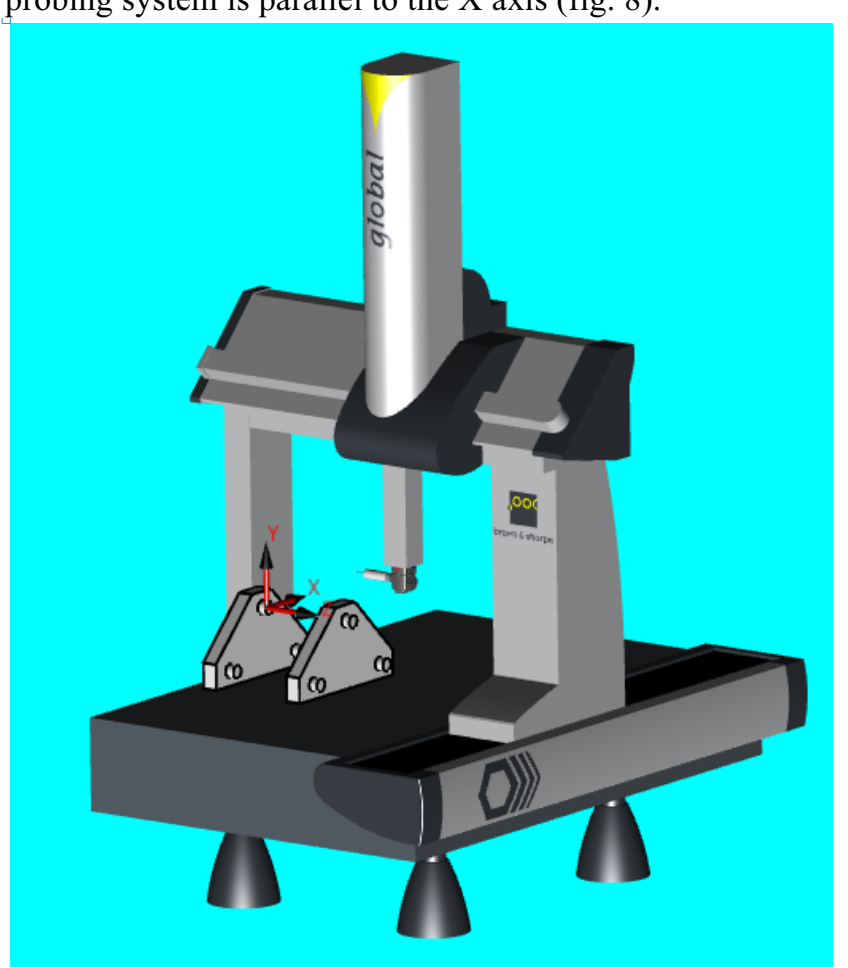

Figure 8: positioning on the $\mathrm{YZ}$ plane

Taking into account the hypotheses made in section 1 , the equation $C_{y}$ becomes:

$$
\begin{gathered}
C_{Y}=L_{X Y}+L_{Y Y}+L_{Z Y}+R_{Y Z}\left(P_{X}+T_{X}\right)-R_{Y X}\left(P_{Z}\right) \\
+R_{X Z} T_{X} \cdot-R_{X X}\left(P_{Z}\right)+R_{Z Z} \cdot T_{X}
\end{gathered}
$$

The distance between two circles aligned along the $\mathrm{Y}$ axis isn't affected by $L_{X Y}, L_{Z Y}, R_{X Z}, R_{X X}$ or $R_{Z Z}(\mathrm{X}$ and $\mathrm{Z}$ values don't change during the measurement), so:

$$
\begin{aligned}
C_{Y 2}-C_{Y 1} & =L_{Y Y}\left\{Y_{2}\right\}-L_{Y Y}\left\{Y_{1}\right\}+\left(R_{Y Z}\left\{Y_{2}\right\}\right. \\
& \left.-R_{Y Z}\left\{Y_{1}\right\}\right) \cdot\left(P_{X}+T_{X}\right)
\end{aligned}
$$

In a second position along the $\mathrm{X}$ axis, parallel to the first, the difference between both measurements is:

$$
\begin{gathered}
\left(C_{Y 2}-C_{Y 1}\right)-\left(C_{Y 4}-C_{Y 3}\right) \\
=\left(R_{Y Z}\left\{Y_{2}\right\}-R_{Y Z}\left\{Y_{1}\right\}\right) \cdot\left(P_{X 2}-P_{X 1}\right)
\end{gathered}
$$

It gives the value of the global $\mathrm{Y}$ yaw $\mathrm{R}_{\mathrm{YZ}}$ on a length of $P_{X 2}-P_{X 1}$

With those equations, $R_{Y Z}$ is known, so the linear defect $\mathrm{L}_{\mathrm{YY}}$ can be calculated.

In those positions, the height of the triangle is along the $\mathrm{Z}$ axis. The equation $C_{Z}$ (compensation along $\mathrm{Z}$ ) becomes:

$$
C_{Z}=L_{X Z}+L_{Y Z}+L_{Z Z}+R_{Y Y}\left(P_{X}+T_{X}\right)-R_{X Y} T_{X}-R_{Z Y} T_{X}
$$

The compensation on the height is:

$$
\begin{gathered}
\left(C_{Z 2}-C_{Z 1}\right)=L_{Z Z}\left\{Z_{2}\right\}-L_{Z Z}\left\{Z_{1}\right\}-\left(R_{Z Y}\left\{Z_{2}\right\}\right. \\
\left.-R_{Z Y}\left\{Z_{1}\right\}\right) T_{X}
\end{gathered}
$$

A third position in the $\mathrm{YZ}$ plane is needed, with a longer tip than the first one. The standard must be moved so that the CMM stays in the same position on three axes.

The difference between both positions is:

$$
\begin{gathered}
\left(C_{Z 2}-C_{Z 1}\right)-\left(C_{Z 4}-C_{Z 3}\right) \\
=\left(R_{Z Y}\left\{Z_{2}\right\}-R_{Z Y}\left\{Z_{1}\right\}\right)\left(T_{X 2}-T_{X 1}\right)
\end{gathered}
$$

It allows calculating the $\mathrm{Z}$ yaw $R_{Z Y}$, so $L_{Z Z}$ can be known.

\subsection{Linear measurements in the ZX plane}

In this application, the standard is still positioned vertically, the hypotenuse along the $\mathrm{X}$ axis. $T_{x}$ and $T_{z}$ are null, $T_{y}$ is the tip offset along Y.

Equation $C_{x}$ becomes:

$$
\begin{gathered}
C_{X}=L_{X X}+L_{Y X}+L_{Z X}+R_{Y Y \cdot}\left(P_{Z}\right)-R_{Y Z} T_{Y}+R_{X Y \cdot}\left(P_{Z}\right) \\
-R_{X Z} T_{Y}-R_{Z Z} T_{Y}
\end{gathered}
$$

The distance between two circles aligned along the $\mathrm{X}$ axis is:

$$
\begin{gathered}
C_{X 2}-C_{X 1}=L_{X X}\left\{X_{2}\right\}-L_{X X}\left\{X_{1}\right\}-\left(R_{X Z}\left\{X_{2}\right\}\right. \\
\left.-R_{X Z}\left\{X_{1}\right\}\right) T_{Y}
\end{gathered}
$$

A second position in the ZX plane is needed, with a longer tip than the first one. The standard must be moved so that the CMM stays in the same position on three axes.

$$
\begin{gathered}
\left(C_{X 2}-C_{X 1}\right)-\left(C_{X 4}-C_{X 3}\right) \\
=-\left(R_{X Z}\left\{X_{2}\right\}-R_{X Z}\left\{X_{1}\right\}\right)\left(T_{Y 2}\right. \\
\left.-T_{Y 1}\right)
\end{gathered}
$$

$L_{X X}$ is known, so the $\mathrm{X}$ yaw $R_{X Z}$ can be calculated directly.

Applying the same method along the $\mathrm{Z}$ axis, $C_{z}$ becomes:

$$
C_{Z}=L_{X Z}+L_{Y Z}+L_{Z Z}+R_{Y X} \cdot T_{Y}-R_{Y Y}\left(P_{X}\right)+R_{X X} \cdot T_{Y}
$$$$
+R_{Z X} \cdot T_{Y}
$$ 
The height of the triangle is compensated by:

SO:

$\left(C_{Z 2}-C_{Z 1}\right)-\left(C_{Z 4}-C_{Z 3}\right)=\left(R_{Z X}\right)\left(T_{Y 2}-T_{Y 1}\right)$

This allows calculating the $Z$ pitch $R_{Z X}$.

\subsection{Measurements along diagonals}

In this chapter, the previous measurements are treated, taking into account all the known results.

\subsubsection{Diagonals in the XY plane}

The compensation along a diagonal in the $\mathrm{XY}$ plane is calculated from $C_{X}$ and $C_{Y}$ equations.

$$
\begin{gathered}
C_{X}=L_{X X}+L_{Y X}+L_{Z X}+R_{Y Y} \cdot\left(P_{Z}+T_{Z}\right)+R_{X Y} \cdot\left(P_{Z}+T_{Z}\right) \\
+R_{Z Y} \cdot T_{Z} \\
C_{Y}=L_{X Y}+L_{Y Y}+L_{Z Y}+R_{Y Z} \cdot P_{X}-R_{Y X}\left(P_{Z}\right. \\
\left.+T_{Z}\right)-R_{X X}\left(P_{Z}+T_{Z}\right)-R_{Z X} T_{Z}
\end{gathered}
$$

The contribution of each axis defect on the distance between two circles $P_{1}$ and $P_{3}$ along a CMM diagonal is:

$$
\begin{gathered}
\left(C_{P 3 X}-C_{P 1 X}\right)=L_{X X}\left\{X_{3}\right\}-L_{X X}\left\{X_{1}\right\}+L_{Y X}\left\{Y_{3}\right\} \\
-L_{Y X}\left\{Y_{1}\right\}+\left(R_{X Y}\left\{X_{3}\right\}-R_{X Y}\left\{X_{1}\right\}\right. \\
\left.+R_{Y Y}\left\{Y_{3}\right\}-R_{Y Y}\left\{Y_{1}\right\}\right) \cdot\left(P_{Z}+T_{Z}\right)
\end{gathered}
$$

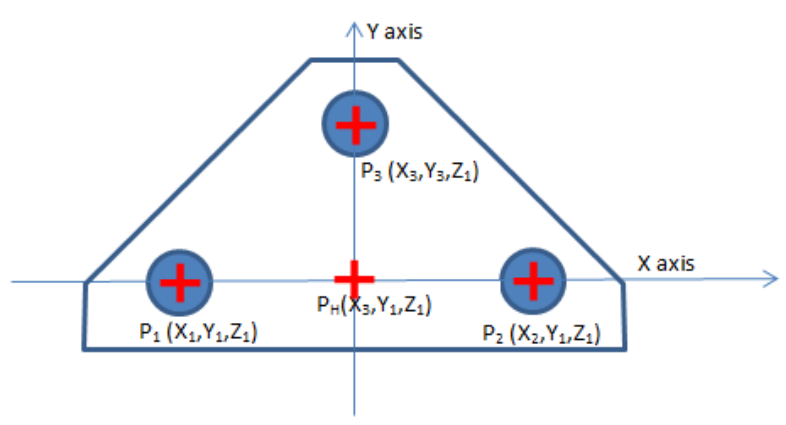

Figure 9: Diagonals in the XY plane

The compensation along diagonals should be calculated from linear compensations, using Chasles relation and Pythagoras. The calculation uses point $\mathrm{P}_{\mathrm{H}}$ (fig. 9), comparing along the $X$ axis the compensation on $P_{1} P_{3}$ and $\mathrm{P}_{1} \mathrm{P}_{\mathrm{H}}$, and along the $\mathrm{Y}$ axis the compensation on $\mathrm{P}_{1} \mathrm{P}_{3}$ and $\mathrm{P}_{\mathrm{H}} \mathrm{P}_{3}$. The difference between both ways of calculation is the effect of a diagonal move.

The $\mathrm{X}$ linear effect subtracts from this result, it gives:

$$
\begin{gathered}
\left(C_{P 3 X}-C_{P 1 X}\right)-\left(C_{P H X}-C_{P 1 X}\right) \\
=L_{Y X}\left\{Y_{3}\right\}-L_{Y X}\left\{Y_{1}\right\}+\left(R_{Y Y}\left\{Y_{3}\right\}-R_{Y Y}\left\{Y_{1}\right\}\right) \cdot\left(P_{Z}+T_{Z}\right)
\end{gathered}
$$

with $\left(C_{P 3 X}-C_{P I X}\right)$ which represents the projection along the $\mathrm{X}$ axis of the measured defect, using the cosine of standard angle.

The same approach on the $\mathrm{Y}$ axis allows writing the defect on the diagonal $\mathrm{P}_{1} \mathrm{P}_{3}$ :

$$
\begin{gathered}
C_{P 3 Y}-C_{P 1 Y}=L_{X Y}\left\{X_{3}\right\}-L_{X Y}\left\{X_{1}\right\}+L_{Y Y}\left\{Y_{3}\right\} \\
-L_{Y Y}\left\{Y_{1}\right\}-\left(R_{Y X}\left\{Y_{3}\right\}-R_{Y X}\left\{Y_{1}\right\}\right. \\
\left.+R_{X X}\left\{X_{3}\right\}-R_{X X}\left\{X_{1}\right\}\right)\left(P_{Z}+T_{Z}\right) \\
+\left(R_{Y Z}\left\{Y_{3}\right\} P_{X 3}-R_{Y Z}\left\{Y_{1}\right\} P_{X 1}\right)
\end{gathered}
$$

Subtracting the linear defect, it gives:

$\left(C_{P 3 Y}-C_{P 1 Y}\right)-\left(C_{P 3 Y}-C_{P H Y}\right)=L_{X Y}\left\{X_{3}\right\}-L_{X Y}\left\{X_{1}\right\}$ $+\left(R_{X X}\left\{X_{3}\right\}-R_{X X}\left\{X_{1}\right\}\right)\left(P_{Z}+T_{Z}\right)+R_{Y Z}\left\{Y_{1}\right\}\left(P_{X 3}-P_{X 1}\right)$

with $\left(C_{P 3 Y}-C_{P 1 Y}\right)$ which represents the projection along the $\mathrm{Y}$ axis of the measured defect, using the sine of standard angle.

Applying the same reasoning on the higher position of the standard, it allows us to discard straightness and the Y yaw defects, which do not depend on the height:

$$
\begin{gathered}
\left(C_{P 6 X}-C_{P 4 X}\right)-\left(C_{P H 2 X}-C_{P 4 X}\right)-\left(C_{P 3 X}-C_{P 1 X}\right) \\
+\left(C_{P H X}-C_{P 1 X}\right) \\
=\left(R_{Y Y}\left\{Y_{3}\right\}-R_{Y Y}\left\{Y_{1}\right\}\right) \cdot\left(Z_{2}-Z_{1}\right)
\end{gathered}
$$

and:

$\left(C_{P 6 Y}-C_{P 4 Y}\right)-\left(C_{P 6 Y}-C_{P H 2 Y}\right)-\left(C_{P 3 Y}-C_{P 1 Y}\right)$

$+\left(C_{P 3 Y}-C_{P H Y}\right)=\left(R_{X X}\left\{X_{3}\right\}-R_{X X}\left\{X_{1}\right\}\right)\left(Z_{2}-Z_{1}\right)$

Those equations describe the displacement effect along a diagonal, and allow calculating the $\mathrm{X}$ and $\mathrm{Y}$ rolls on a length of respectively $\mathrm{P}_{1} \mathrm{P}_{\mathrm{H}}$ and $\mathrm{P}_{\mathrm{H}} \mathrm{P}_{3}$. Even if they can be calculated, straightness defects $L_{X Y}$ and $L_{Y X}$ represent only local values, which take into account a part of the perpendicularity. For those reasons, the result is not accurate enough to be described.

\subsubsection{Diagonals in the ZX plane}

The compensation along a diagonal in the $\mathrm{YZ}$ plane is calculated from $C_{x}$ :

$$
\begin{gathered}
C_{X}=L_{X X}+L_{Y X}+L_{Z X}+R_{Y Y} \cdot\left(P_{Z}\right) \\
-R_{Y Z} T_{Y}+R_{X Y} \cdot\left(P_{Z}\right)-R_{X Z} T_{Y}-R_{Z Z} T_{Y}
\end{gathered}
$$

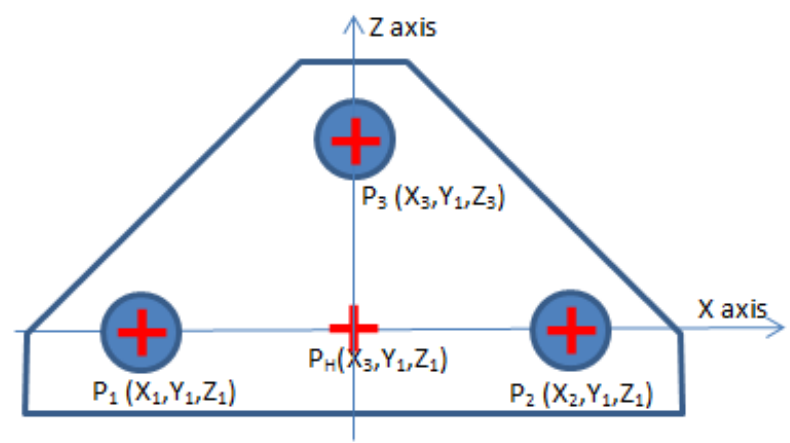

Figure 10: Diagonals in the ZX plane

The compensation on $P_{1} P_{3}$ in the $\mathrm{ZX}$ plane (fig. 10) can be written:

$$
C_{P X 3}-C_{P X 1}=L_{X X}\left\{X_{3}\right\}-L_{X X}\left\{X_{1}\right\}+L_{Z X}\left\{Z_{3}\right\}
$$

$-L_{Z X}\left\{Z_{1}\right\}-\left(R_{X Z}\left\{X_{3}\right\}-R_{X Z}\left\{X_{1}\right\}\right) T_{Y}-\left(R_{Z Z}\left\{Z_{3}\right\}-R_{Z Z}\left\{Z_{1}\right\}\right) T_{Y}$ $+\left(R_{Y Y}\left\{Y_{1}\right\}+R_{X Y}\left\{X_{3}\right\}-R_{X Y}\left\{X_{1}\right\}\right)\left(Z_{3}-Z_{1}\right)$ 
The linear effect on $P_{l} P_{H}$ is:

$$
\begin{gathered}
C_{P X H}-C_{P X 1}=L_{X X}\left\{X_{3}\right\} \\
-L_{X X}\left\{X_{1}\right\}-\left(R_{X Z}\left\{X_{3}\right\}-R_{X Z}\left\{X_{1}\right\}\right) T_{Y}
\end{gathered}
$$

The difference between both equations gives:

$$
\begin{gathered}
\left(C_{P X 3}-C_{P X 1}\right)-\left(C_{P X H}-C_{P X 1}\right) \\
=L_{Z X}\left\{Z_{3}\right\}-L_{Z X}\left\{Z_{1}\right\}-\left(R_{Z Z}\left\{Z_{3}\right\}-R_{Z Z}\left\{Z_{1}\right\}\right) T_{Y} \\
+\left(R_{Y Y}\left\{Y_{1}\right\}+R_{X Y}\left\{X_{3}\right\}-R_{X Y}\left\{X_{1}\right\}\right)\left(Z_{3}-Z_{1}\right)
\end{gathered}
$$

The same calculation in the second position in the ZX plane allows calculating the $\mathrm{Z}$ roll $R_{Z Z}$ :

$$
\begin{gathered}
\left(C_{P X 6}-C_{P X 4}\right)-\left(C_{P X H 2}-C_{P X 4}\right)-\left(C_{P X 3}-C_{P X 1}\right) \\
+\left(C_{P X H}-C_{P X 1}\right) \\
=\left(R_{Z Z}\left\{Z_{3}\right\}-R_{Z Z}\left\{Z_{1}\right\}\right)\left(T_{Y 1}-T_{Y 2}\right)
\end{gathered}
$$

All the local straightness defects can be calculated with the same method, without any additional position.

\subsubsection{Perpendicularity between axes}

The calculation of perpendicularity between axes, two by two, is done from difference between the measured lengths of the triangle sides.

The product of the length of a side by the cosine of the real angle of perpendicularity should be equal to the same product on the other side (fig. 11).

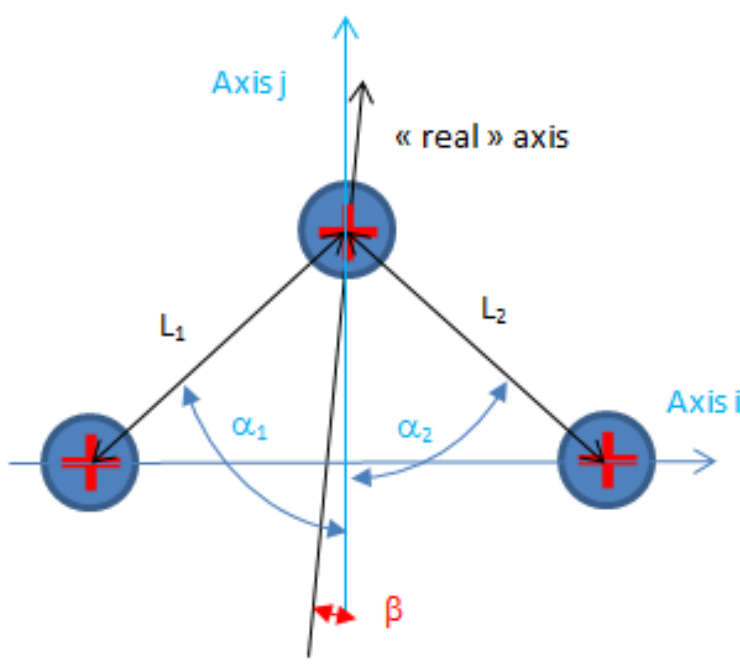

Figure 11: perpendicularity calculation

It can be written like this:

\begin{tabular}{|ll}
\hline & $L_{1} \cdot \cos \left(\alpha_{1}-\beta\right)=L_{2} \cdot \cos \left(\alpha_{2}+\beta\right)$ \\
& $L_{1} \cdot\left(\cos \alpha_{1} \cos \beta+\sin \alpha_{1} \sin \beta\right)$ \\
& $=L_{2} \cdot\left(\cos \alpha_{2} \cos \beta-\sin \alpha_{2} \sin \beta\right)$ \\
& $\cos \beta\left(L_{1} \cos \alpha_{1}-L_{2} \cos \alpha_{2}\right)$ \\
& $=-\sin \beta\left(L_{1} \sin \alpha_{1}+L_{2} \sin \alpha_{2}\right)$ \\
\hline So: & $\tan \beta=\frac{L_{2} \cos \alpha_{2}-L_{1} \cos \alpha_{1}}{L_{1} \sin \alpha_{1}+L_{2} \sin \alpha_{2}}$ \\
&
\end{tabular}

$\beta$ is the perpendicularity defect between two axes at a given position.

\section{Real case example}

As described previously, the standard can be measured horizontally or vertically (fig. 12). The global checking proposed here needs only 7 different positions.

The standard is considered as a perfect part (uncertainties known), so the defects are affected to the CMM geometry.

The measured lengths and angles are exported in a spreadsheet, and compared to the theoretical values. It's also useful to export tip offsets, and diameter of each measured circle. The length deviations are assimilated to residual compensation defects, so that each parameter can be calculated with previous equations (chapter. 3 ).

The total measurement ( 7 positions, 3 repetitions) takes less than 1 hour, instead of 2 hours for ISO10-360-2 test, or 1 hour for ball bar test, which don't point out the bad parameters. Automation allows treating results directly, so that the bad parameter(s) can be corrected quickly. A partial control, which gives only a picture of linear defects and perpendicularities, can be done in few minutes. It allows checking parameters that are most sensitives.

A tolerance has to be defined for each parameter to point out any abnormal deviation. This tolerance should depend on the CMM type, the probe, the stylus...

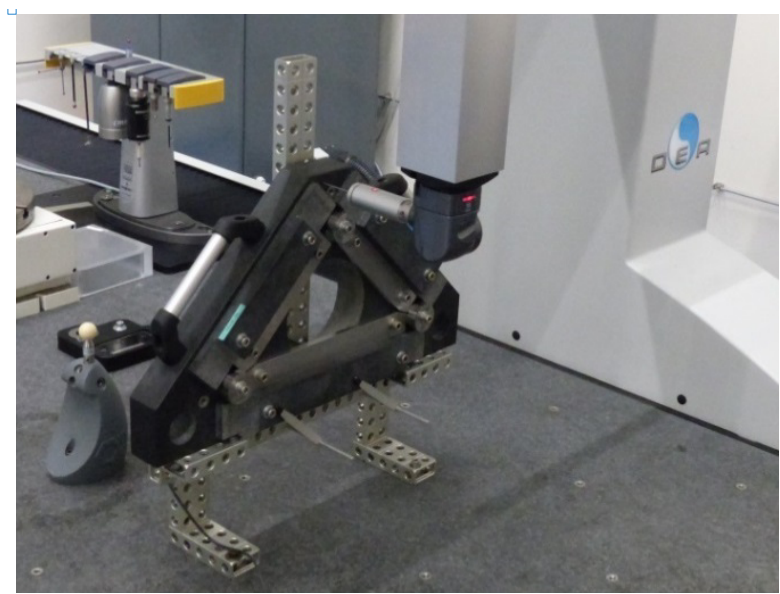

Figure 12: standard vertically positioned (ZX plane)

Usually, the length deviation average (3 lengths, 7 positions, 3 repetitions) should be close to zero, the standard deviation close to standard uncertainty.

\section{Conclusion}

This new standard allows a fast checking of the global CMM geometry, with results linked to metrological definition of the meter. Furthermore, in case of defects, the standard permits defining the bad parameters.

All the measurements give also information about uncertainty on lengths and angles on the CMM.

The assembly is easy to do, and the uncertainty caused by it is negligible, if it's done properly. This study had been done with a prototype. Other sizes or forms are 
described in the patent, and allows optimizing the checking for different CMMs.

Using this new tool is a good way to perform an effective traceability of a CMM geometrical quality.

\section{References}

[1] ISO 10360-2:2009, Geometrical product specifications (GPS), Acceptance and reverification tests for coordinates measuring machines (CMM), part 2 : CMMs used for measuring linear dimensions.

[2] ANSI B89.4.1-1997 specification, Methods for performance evaluation of coordinate measuring machines.

[3] The Machine Checking Gauge (MCG), Renishaw, Patents EP 0180610 and US 4777818.

[4] P. Bourdet, Contribution à la mesure tridimensionnelle, Thesis / PhD (1987).

[5] L'accréditation des mesures tridimensionnelles Collège Français de Métrologie (2008).

[6] Two-dimensional Metrological Standard, CEA, Patents EP2725231 and US 2014/0109646. 